\title{
Elimination d'un prophage dans des souches mono- et multilysogènes de streptocoques du groupe $\mathbf{N}$
}

\author{
par \\ Marie-Christine CHOPIN*, Annette ROUAULT* \\ et Micheline ROUSSEAU***
}

\section{R é s u m é}

En utilisant l'action combinée de la mitomycine $\mathrm{C}$ et des ultraviolets nous avons guéri 4 souches lysogènes de streptocoques lactiques sur les 12 testées. Deux de ces souches ne sont plus inductibles et semblent guéries de tout prophage. Les deux autres, multilysogènes, n'ont été guéries que d'un prophage dont la perte s'est accompagnée de changements d'immunité ou de sensibilité aux phages. Aucune modification de la vitesse d'acidification des souches cultivées sur lait n'a été associée à la perte d'un prophage.

Titre abrégé :

Guérison de Streptocoques lactiques lysogènes.

Mots clés :

Streptococcus lactis - Streptococcus lactis subsp. diacetylactis - Streptococcus cremoris - Lysogènie - Prophage - Guérison.

\section{S u m m a ry}

Four lactic streptococci strains out of 12 were cured of their prophage by using mitomycin $C$ and ultraviolet light. After treatment two of them were no more inducible. The two others, multilysogenic, were only cured from one prophage. Their immunity or their quantitative aptitude to allow phages development was modified

Institut National de la Recherche Agronomique.

* Laboratoire de Recherches de Technologie Laitière, 65, rue de Saint-Brieuc 35042 Rennes cedex.

** Laboratoire de Microbiologie Laitière, C.N.R.Z. - 78350 Jouy-en-Josas. 
by loss of one prophage. Acidification rate of strains in milk was not modified by prophage loss.

Title:

Prophage curing of mono- and mutilysogenic group N streptococcal strains.

Key words:

Streptococcus lactis - Streptococcus lactis subsp. diacetylactis - Streptococcus cremoris - Lysogeny - Prophage - Cure.

\section{INTRODUCTION}

Il est maintenant bien établi que la plupart des souches des streptocoques lactiques sont lysogènes (Kozak et al., 1973; Mc Kay et Baldwin, 1973 ; Lowrie, 1974 ; Lawrence, Thomas et Terzaghi, 1976; Huggins et Sandine, 1977; Davies et Gasson, 1980 ; Reyrolle et al., 1982 ) et que les phages tempérés qu'elles libèrent peuvent être une importante source de contamination des fromageries (Heap et Lawrence, 1977; Lawrence et al., 1978; Reyrolle et al., 1982).

D'après les travaux réalisés chez d'autres espèces, on sait que les phages tempérés présents dans une bactérie lysogène (prophages) peuvent conférer à celle-ci un certain nombre de propriétés. La plus répandue est l'immunité vis-à-vis d'autres phages. Mais d'autres caractères peuvent aussi être liés à la présence d'un prophage comme par exemple, l'état de surface de la bactérie (Holloway et Cooper, 1962), la production d'enzymes (Duval-Iflah, 1972) ou de toxines (Freeman, 1951), la sporulation (Bramucci, Keggins et Lovett, 1977), l'existence de systèmes de restriction/modification (Glover et al., 1963; Trautner et al., 1980). Chez les bactéries lactiques, les caractères associés aux prophages ne sont pas connus. La première étape pour les connaître est l'obtention de souches guéries de ces prophages. Mais la guérison des streptocoques lactiques semble difficile. Gasson et Davies (1980) d'une part et Georghiou, Phua et Terzaghi (1981) d'autre part sont parvenus récemment à isoler des clones spontanément guéris après élimination d'une fraction de la population lysogène par induction aux ultraviolets. Mais 3 souches seulement ont été guéries de cette manière. Les 2 équipes ont guéri une même souche de Streptococcus cremoris R1 et 2 souches de Streptococcus lactis ont été guéries par Gasson et Davies (1980). Dans aucun de ces cas une perte des caractères étudiés n'a été associée à la perte d'un prophage.

Cependant, il est probable que l'état lysogène influe sur le déterminisme de certains caractères des streptocoques lactiques. Et connaitre le rôle des prophages pourrait offrir un intérêt dans la sélection des souches et le contrôle des infections de phages en laiterie.

Nous avons donc essayé de guérir 11 souches lysogènes de notre collection. Nous avons ajouté à cette liste une souche de 
S. cremoris R1 à des fins de comparaison avec les deux autres travaux réalisés dans ce domaine (Gasson et Davies, 1980 ; Georghiou, Phua et Terzaghi, 1981). Ce travail nous a amenés à améliorer la méthode décrite par Gasson et Davies (1980). Nous avons cependant rencontré des difficultés certainement liées à l'état multilysogène qui semble fréquent dans nos souches. Pour 2 des 4 souches guéries, des changements de sensibilité aux phages ont été observés.

\title{
MATERIEL ET METHODES
}

\section{Souches bactériennes et bactériophages}

Nous avons utilisé 12 souches lysogènes de notre collection (tab. 1). Ces souches ont été choisies dans les groupes lytiques G2,

\author{
TABLEAU 1 - TABLE 1
}

Souches utilisées - Strains used

\begin{tabular}{|c|c|}
\hline Souches & Origine et propriétés \\
\hline $\begin{array}{l}\text { S. cremoris } \\
\text { TL16 } \\
\\
\text { TL413 } \\
\text { TL788 } \\
\text { TL1314 }\end{array}$ & $\begin{array}{l}\text { Z107 (Cl), souche reçue du Commonwealth. } \\
\text { Scientific and Industrial Research } \\
\text { Organisation (C.S.I.R.O., Australie, 1958). } \\
\text { A15, souche isolée de lait cru. } \\
\text { Z114 (R1), souche reçue du C.S.I.R.O. (1958). } \\
\text { TL788 guérie de son prophage. }\end{array}$ \\
\hline $\begin{array}{l}\text { S. lactis } \\
\text { TL13 } \\
\text { TL414 } \\
\text { TL562 } \\
\text { TL1343 } \\
\text { TL823 } \\
\\
\text { TL1342 } \\
\text { TL1333 } \\
\text { TL929 } \\
\text { TL933 } \\
\text { TL1359 } \\
\text { TL1360 } \\
\text { TL583 }\end{array}$ & $\begin{array}{l}\text { Z261, origine inconnue. } \\
\text { A44, souche isolée de lait cru. } \\
\text { A100, origine inconnue. } \\
\text { TL562 guérie de son prophage. } \\
\text { Z167, souche reçue du Laboratory of Pure } \\
\text { Dairy Cultures (Olsztyn - Pologne). } \\
\text { Multilysogène. } \\
\text { TL823 guérie d'un prophage. } \\
\text { TL1342 relysogénisée. } \\
\text { A57, souche isolée d'un levain commercial. } \\
\text { A61, souche isolée de lait cru. } \\
\text { Multilysogène. } \\
\text { TL933 guérie d'un prophage. } \\
\text { TL1359 relysogènisée. } \\
\text { Souche isolée d'un levain commercial. }\end{array}$ \\
\hline $\begin{array}{l}\text { S. lactis subsp. } \\
\text { diacetylactis } \\
\text { TL427 } \\
\text { TL925 }\end{array}$ & $\begin{array}{l}\text { A83, souche isolée d'un levain commercial. } \\
\text { A50, souche isolée d'un levain commercial. }\end{array}$ \\
\hline
\end{tabular}


G3, G4 et G5 définis lors d'un précédent travail d'après le spectre de sensibilité de 291 souches à 132 phages virulents (Chopin, Chopin et Roux, 1976). La souche $S$. cremoris TL788 (R1) sensible à un seul phage de notre collection n'avait pu être rattachée à aucun des groupes lytiques déterminés. Ces souches sont toutes inductibles aux ultraviolets (UV) et à la mitomycine C (MC). Nous disposons de souches indicatrices des phages tempérés libérés par 6 d'entre elles.

Les phages virulents utilisés ont été décrits précédemment (Chopin, Chopin et Roux, 1976). Les phages tempérés ont été obtenus par induction de souches bactériennes appartenant à notre collection.

\section{Induction des souches aux UV}

Des cultures de $18 \mathrm{~h}$ étaient diluées à $2 \%$ dans $20 \mathrm{ml}$ de M17 (Terzaghi et Sandine, 1975) et incubées 1 h 30 à 2 h à $30^{\circ} \mathrm{C}$ (début de phase exponentielle de croissance). Après centrifugation (3000 g$10 \mathrm{~min}$ ), les culots étaient remis en suspension dans $20 \mathrm{ml}$ de Ringer dilué au quart. Quinze $\mathrm{ml}$ étaient irradiés dans des boîtes de Petri de $95 \mathrm{~mm}$ de diamètre, avec agitation, durant 5 secondes avec une lampe General Electric (2537 A) située à $40 \mathrm{~cm}\left(270\right.$ ergs. $\left.\mathrm{mm}^{-2}\right)$ et $5 \mathrm{ml}$ non irradiés servaient de témoin. Quinze $\mathrm{ml}$ de M17 double concentration étaient ajoutés à la culture irradiée et $5 \mathrm{ml}$ au témoin. L'incubation était prolongée à $30^{\circ} \mathrm{C}$, à l'obscurité, jusqu'à éclaircissement de la culture irradiée. L'absorbance (A) des cultures témoin et induite était par ailleurs suivie en continu avec un biophotomètre (Bonet-Maury-Jouan, France).

\section{Induction des souches à la MC}

La technique utilisée a été décrite précédemment (Reyrolle et al., 1982). Les cultures en début de phase exponentielle de croissance étaient induites par addition de $\mathrm{MC}$ à la concentration finale de $1 \mu \mathrm{g} / \mathrm{ml}$ et incubées à $30^{\circ} \mathrm{C}$. Après éclaircissement des cultures induites par rapport au témoin, les lysats étaient filtrés sur des membranes Millipore (HA $0,45 \mu \mathrm{m}$ ).

\section{Microscopie électronique}

Les lysats obtenus après induction des souches aux UV ou à la MC étaient centrifugés à $12000 \mathrm{~g}$ pendant $10 \mathrm{~min}$ pour éliminer les débris cellulaires. Les phages étaient ensuite sédimentés pendant $3 \mathrm{~h}$ à $48000 \mathrm{~g}$. Les culots étaient laissés en contact avec $1 \mathrm{ml}$ d'acétate d'ammonium $0,1 \mathrm{M}$ à $4^{\circ} \mathrm{C}$ pendant une nuit avant d'être remis en suspension. Les grilles recouvertes de collodion carboné étaient chargées par une technique de filtration sur gélose (Anderson et Doane, 1972). Les préparations étaient colorées avec de l'acide phosphotungstique à pH 7,2. Les observations étaient faites avec un microscope Zeiss EM 10A. 


\section{Guérison des souches et relysogènisation}

Nous avons utilisé la technique décrite par Gasson et Davies (1980) et une modification de cette technique utilisant à la fois la MC et les UV : une culture d'une nuit sur M17 était ensemencée à $2 \%$ sur bouillon M17 additionné de MC. La concentration finale en MC utilisée était la plus élevée entre 0,1 et $1 \mu \mathrm{g} \cdot \mathrm{ml}^{-1}$ permettant une croissance normale de la culture en $18 \mathrm{~h}$ à $30^{\circ} \mathrm{C}$. La culture était ensuite repiquée sur bouillon M17, et incubée 2 h 30 à $30^{\circ} \mathrm{C}$, avant d'être étalée à différentes dilutions sur M17 gélosé et induite aux U.V. Les clones guéris étaient sélectionnés et isolés selon la technique décrite par Gasson et Davies (1980). Cent clones au moins étaient remis en suspension dans $0,2 \mathrm{ml}$ de M17-10\% et déposés en gouttes sur deux plaques de M17 contenant 0,01 M de chlorure de calcium. L'une des plaques était inondée avec le lysat UV ou MC de la souche lysogène de départ. L'autre servait de témoin. Les clones présentant des plages de lyse étaient réisolés 2 fois sur M17 gélosé. On vérifiait qu'ils n'étaient plus inductibles et étaient devenus sensibles aux phages libérés par la souche lysogène d'origine. Leur guérison était confirmée par relysogénisation par le phage homologue : les bactéries provenant du centre d'une zone de lyse étaient réisolées 2 fois sur M17 gélosé et des colonies étaient testées pour leur sensibilité au phage.

L'inductibilité des clones résistant au phage était vérifiée en suivant l'absorbance des cultures en continu et le spectre lytique des phages tempérés libérés était contrôlé.

Les numéros des souches lysogènes, guéries et relysogénisées sont rassemblés dans le tableau 1 .

\section{RESULTATS}

\section{Guérison des souches}

En utilisant la technique décrite par Gasson et Davies (1980) nous avons obtenu $14 \%$ de clones guéris parmi les survivants à l'irradiation UV de $S$. cremoris TL788 (R1). Nous n'avons pu guérir aucune des 11 autres souches testées en utilisant cette technique.

En utilisant l'action combinée de la MC et des UV, nous avons obtenu $30 \%$ de clones guéris pour S. cremoris TL788 (R1). Nous avons en outre guéri la souche S. lactis TL562 (6\%). Les clones guéris obtenus pour ces deux souches ne sont plus inductibles par la MC ou les UV, c'est-à-dire que l'absorbance des cultures ne chute pas et il n'y a plus libération de phages actifs sur les souches indicatrices.

L'action combinée de la MC et des UV nous a également permis de guérir les souches TL823 (2\%) et TL933 (1,5\%). Mais les clones 
guéris obtenus, sensibles au lysat UV de la souche de départ, étaient encore inductibles laissant supposer que ces souches sont multilysogènes. En effet, l'examen en microscopie électronique des lysats obtenus par induction de ces 2 souches avant guérison a mis en évidence la présence de plusieurs types morphologiques de phages. La souche TL823 libère au moins 2 phages à tête isométrique de dimensions similaires différant par la longueur de la queue, la présence ou l'absence d'un collier et d'une fibre caudale (fig. 1 a-b).

a et $\mathrm{b}$ : phages tempérés isométriques libérés par la souche TL823.

a : phage présentant un collier et une plaque basale prolongée par une fibre.

b : phage présentant une plaque basale à crochets.

c : phages tempérés isométriques et prolates libérés par la souche TL933.

Les barres indiquent $100 \mathrm{~nm}$.

$a$ and $b$ : isometric temperate phages induced from strain TL823.

a: phage with a collar and a long tail fiber originating from a small baseplate.

b: phage with prongs on the baseplate.

$c:$ isometric and prolate temperate phages induced from strain TL933.

Bars represent $100 \mathrm{~nm}$.
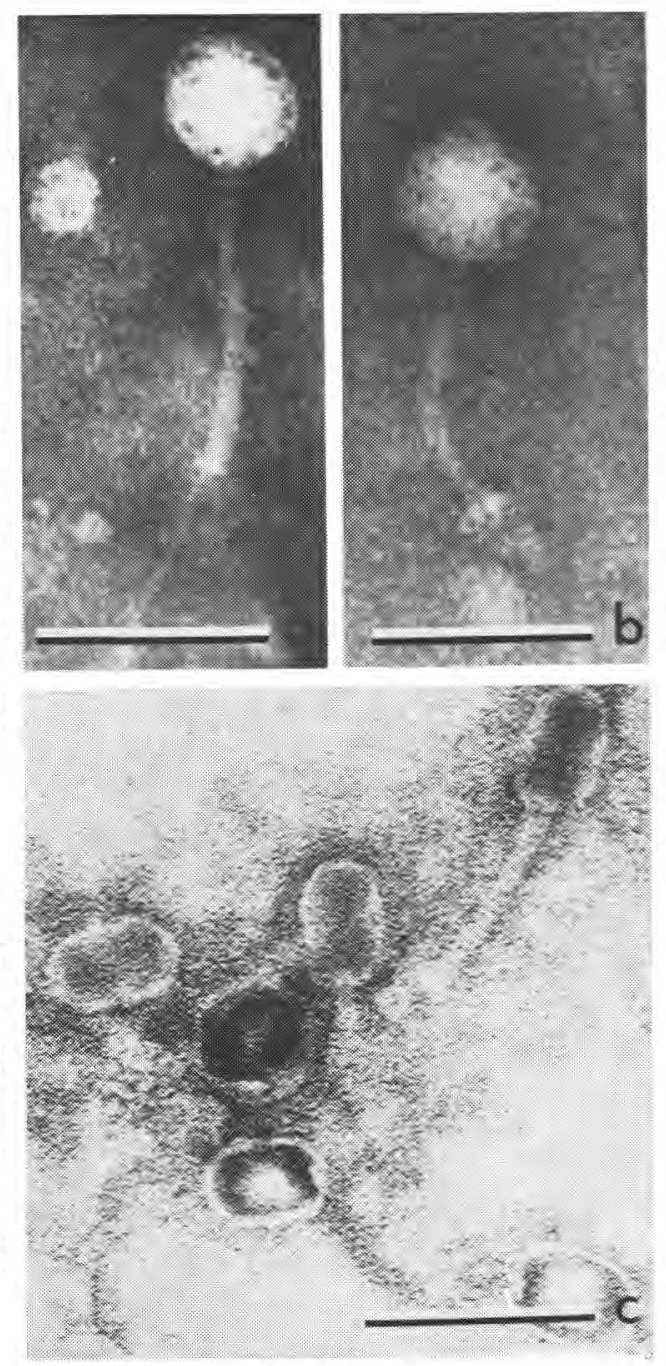

Fig. 1 
La souche TL933 libère 1 phage à tête isométrique et un autre à tête prolate. Nous avons observé quelques phages prolates entiers mais surtout de nombreuses têtes isolées vides ou partiellement vidées dont certaines présentaient des structures anormales ressemblant aux structures tubulaires du phage lambda (Kemp, Howatson et Siminovitch, 1968). De la même façon, les phages isométriques entiers étaient rares et les têtes isolées vides ou partiellement vidées nombreuses. Les proportions relatives de ces 2 types de phages varient en fonction du type d'induction utilisé (UV ou MC). Les lysats obtenus par induction de TL933 aux UV révèlent des phages à tête isométrique alors que ceux obtenus par induction à la MC montrent essentiellement des phages à tête prolate et peu de phages à tête isométrique (fig. 1-c).

L'examen du lysat obtenu par induction de la souche TL823 guérie semble indiquer que le phage présentant une fibre caudale a été éliminé. Après induction de la souche TL933 guérie, seule la forme isométrique a été observée.

Les 8 autres souches testées n'ont pas pu être guéries par la technique utilisée. L'observation des lysats obtenus après induction aux UV de 2 de ces souches (TL925 et TL583) a révélé la présence de 2 phages de morphologies différentes dans chacun d'eux. Ces phages sont à tête isométrique et diffèrent par la longueur de leur queue et les structures terminales (fig. 2).

\section{Influence de la perte d'un prophage sur la croissance des souches}

Nous n'avons noté aucune différence dans la croissance sur M17 des souches lysogènes et des souches guéries. Il en a été de même pour les vitesses d'acidification sur lait, à l'exception toutefois de la souche guérie TL1342 qui acidifie plus lentement le lait que la souche lysogène de départ. Mais l'activité acidifiante de cette souche n'est pas restaurée après sa relysogénisation par le phage homologue.

\section{Modification du spectre de sensibilité aux phages des souches guéries d'un prophage}

La perte d'un prophage ne modifie pas le spectre de sensibilité aux phages virulents des souches TL788, TL823 et TL562. Pour aucune de ces souches guéries nous n'avons noté l'apparition d'une sensibilité à l'un des phages test choisis pour représenter les différents groupes lytiques de notre collection. Les souches TL562 et TL823 appartiennent à un même groupe lytique (G3). Ce groupe rassemble des souches sensibles à un groupe correspondant de 63 phages. La guérison de TL562 et TL823 ne modifie pas leur spectre de sensibilité à ces 63 phages.

Cependant, la souche TL823 indicatrice des phages tempérés libérés par 5 souches lysogènes de son groupe lytique est plus 
$a$ et $b$ : phages tempérés isométriques libérés par la souche TL583.

a : phage présentant une fibre dans le prolongement de la queue.

b : phage présentant une plaque basale à crochets avec une fibre centrale.

c et d: phages tempérés isométriques libérés par la souche TL925. Les 2 phages présentent une plaque basale prolongée par une fibre.

Les barres indiquent 100 $\mathrm{nm}$.

$a$ and $b$ : isometric temperate phages induced from strain 583.

a: phage with a long tail fiber.

$b$ : phage with prongs and a middle fiber on the $b a$ seplate.

$c$ and $d$ : isometric temperate phages induced from strain TL925. Phages have a sinall baseplate with a long fiber.

Bars represent $100 \mathrm{~nm}$.
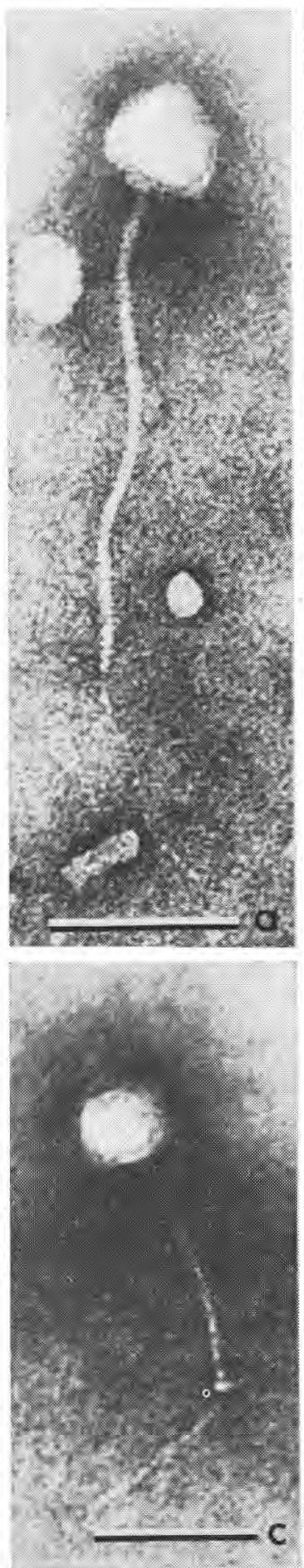

Fig. 2

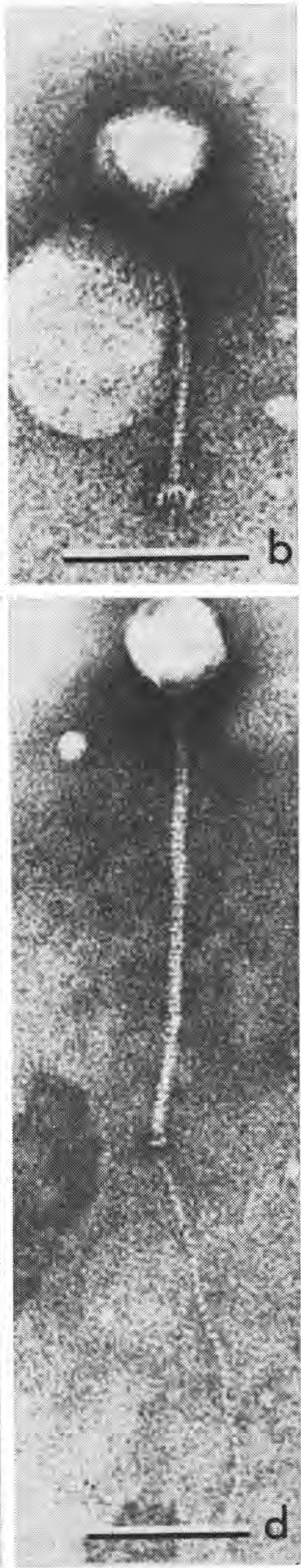


sensible à ces phages lorsqu'elle est guérie (tab. 2). Le nombre de phages dénombrés sur la souche guérie est supérieur de 1 à $2 \log$ à celui obtenu sur la souche lysogène ou sur la souche guérie relysogénisée. Ces observations demeurent vraies après la propagation des phages tempérés sur la souche lysogène TL823.

Par contre, la perte du phage prolate rend TL933 sensible à tous les phages prolates virulents de son groupe lytique (tab. 3). Cette souche devient également sensible aux phages tempérés libérés par trois souches lysogènes de son groupe.

\section{DISCUSSION}

En utilisant la technique de guérison décrite par Gasson et Davies (1980), nous avons obtenu 14\% de clones guéris avec la souche $S$. cremoris TL788 (R1). Ceci représente un pourcentage intermédiaire entre celui de $2 \%$ obtenu par Georghiou, Phua et Terzaghi (1981) et celui de $38 \%$ obtenu par Gasson et Davies (1980) sur une souche de même origine. Cependant, cette technique s'est révélée inefficace sur les 11 autres souches testées. En utilisant I'action combinée de la MC et des UV nous avons pu isoler des clones guéris pour 3 autres souches. Ceci n'est pas étonnant dans la mesure où les mécanismes d'induction par ces 2 agents sont différents. Dans le cas de la souche TL933 cela se traduit par la libération des phages qu'elle héberge dans des proportions différentes selon le mode d'induction utilisé. Mais, cette double action de la MC et des UV augmente les risques de mutation ou de perte de plasmides. C'est vraisemblablement le cas du clone guéri TL1342 étudié qui assimile le lactose et se développe normalement sur M17 mais a perdu la faculté d'acidifier rapidement le lait. Cela suggère que ce clone a dû perdre son activité protéasique indispensable pour assurer une production rapide d'acide sur lait (Mc Kay et Baldwin, 1974). Cette activité n'est pas restaurée après sa relysogènisation par le phage homologue.

Par ailleurs, les seules souches guéries qui ne soient plus inductibles (TL788 (R1) et TL562) semblent monolysogènes. Dans le cas de la souche R1, cette hypothèse est en accord avec les observations réalisées par Gasson et Davies (1980) et Georghiou, Phua et Terzaghi (1981). Sur les 4 souches multilysogènes mises en évidence, 2 n'ont pas pu être guéries et les 2 autres l'ont été d'un seul phage. La difficulté rencontrée pour guérir les souches semble donc liée au moins pour un certain nombre d'entre elles, à l'état multilysogène. A notre connaissance, la présence de 2 phages morphologiquement diférents dans le lysat d'une souche lysogène de streptocoques lactiques a seulement été signalée dans une communication au Congrès de Laiterie de Moscou (Jarvis, 1982). Passant en revue les articles contradictoires relatifs aux phages tempérés portés par 
TABLEAU 2 - TABLE 2

Sensibilité comparée des souches TL823, TL1342 et TL1333 aux phages tempérés libérés par induction aux UV de 4 souches de $S$. lactis subsp. diacetylactis

Lytic sensitivity of strains TL823, TL1342 and TL 1333 to UV-induced temperate phages from 4

S. lactis subsp. diacetylactis strains

\begin{tabular}{|c|c|c|c|c|}
\hline \multirow{2}{*}{ Souches indicatrices } & \multicolumn{2}{|c|}{ Phages tempérés } & \multicolumn{2}{|c|}{ (nombre/ml de lysat) } \\
\hline & ФTL925 & ФTL982 & ФTL985 & ФTL573 \\
\hline TL823 (multilysogène) & $3,0 \times 10^{3}$ & $1,0 \times 10^{4}$ & $3,0 \times 10^{5}$ & $3,0 \times 10^{5}$ \\
\hline TL1342 (guérie d'un prophage) & $5,0 \times 10^{4}$ & $6,2 \times 10^{7}$ & $3,2 \times 10^{7}$ & $2,0 \times 10^{7}$ \\
\hline TL1333 (guérie relysogénisée) & $3,0 \times 10^{3}$ & $2,0 \times 10^{5}$ & $2,0 \times 10^{5}$ & $2,0 \times 10^{5}$ \\
\hline
\end{tabular}


TABLEAU $3-T A B L E 3$

Spectre de sensibilité à des phages virulents et tempérés des souches TL933, TL1359 et TL1360

Sensitivity pattern of strains TL933, TL1359 and TL1360 to virulent and temperate phages

\begin{tabular}{|c|c|c|c|c|c|c|c|c|c|c|c|}
\hline \multirow{2}{*}{ Souches indicatrices } & \multicolumn{8}{|c|}{ Phages virulents } & \multicolumn{3}{|c|}{ Phages tempérés } \\
\hline & $\begin{array}{llcl}6 & 12 & 15 & 22 \\
\mathrm{P}(1) \mathrm{P} & \mathrm{I}(2) \mathrm{I}\end{array}$ & $\begin{array}{l}76 \\
I\end{array}$ & $\begin{array}{cc}27 & 68 \\
\mathrm{I} & \mathrm{I}\end{array}$ & $\begin{array}{lc}8 & 140 \\
{[} & I\end{array}$ & $\begin{array}{l}54 \\
\mathrm{P}\end{array}$ & $\begin{array}{r}16 \\
P\end{array}$ & $\begin{array}{c}168 \\
\mathrm{I}\end{array}$ & $\stackrel{155}{I}$ & $\stackrel{\Phi}{\text { TL790 }}$ & $\underset{\text { TL739 }}{\Phi}$ & $\stackrel{\Phi}{\text { TL960 }}$ \\
\hline TL933 (bilysogène) & ---- & - & - & - & - & + & - & - & - & - & - \\
\hline $\begin{array}{c}\text { TL1359 (guérie d'un } \\
\text { prophage) }\end{array}$ & ++-- & - & - & - & + & + & - & - & + & + & + \\
\hline $\begin{array}{l}\text { TL1360 (guérie } \\
\text { relysogénisée) }\end{array}$ & ---- & - & - & - - & - & + & - & - & - & - & - \\
\hline
\end{tabular}

(1) : P : phage à tête prolate - Prolate phage.

(2) : I : phage à tête isométrique - Isometric phage. 
la souche $S$. lactis C2, Lawrence, Thomas et Terzaghi (1976) avaient suggéré que cette souche pourrait être multilysogène. Mais, cette hypothèse n'a pas été confirmée par l'étude récente de Davies, Underwood et Gasson (1981) qui ont mis en évidence un seul type de phage dans le lysat de cette souche.

La souche TL933 constitue un cas à part. Bilysogène, elle porte 2 phages très différents morphologiquement un à tête prolate et un autre à tête isométrique. La maturation de ces phages doit souvent être incomplète si l'on en juge par la forte proportion de têtes isolées que nous avons observées. Par ailleurs, dans le cas du phage prolate, la synthèse de la tête est altérée donnant des formes tubulaires du type de celles observées chez les phages virulents de même morphologie (Chopin et Rousseau, 1983). Les phages tempérés prolates semblent peu abondants chez les streptocoques lactiques. La majorité des phages tempérés observés sont isométriques (Mc Kay et Baldwin, 1973; Lowrie, 1974; Huggins et Sandine, 1977 ; Gasson et Davies, 1980, Terzaghi et Sandine, 1981). Seuls Heap et Jarvis (1980) ont décrit 3 phages prolates sur les 64 phages tempérés qu'ils ont observés. Ce type de phage semble moins stable dans la souche TL933 que le type isométrique et s'élimine plus facilement.

La souche TL933 est la seule pour laquelle nous avons noté un changement d'immunité à la suite de la perte d'un prophage. En effet, pour les 3 autres souches guéries nous n'avons pas noté de changement dans le spectre de sensibilité aux phages de notre collection.

Par contre, la guérison de la souche TL823 augmente sa sensibilité à 4 phages tempérés. Nos résultats montrent que le prophage limite le développement des autres phages tempérés mais qu'il ne s'agit pas d'un système de restriction/modification. On peut penser qu'il modifie l'état de surface de la bactérie et par conséquent le mécanisme d'adsorption des phages tempérés étudiés ou bien qu'il interfère avec le développement intracellulaire de ces phages comme cela a été observé chez Bacillus subtilis (Rettenmier et Hemphill, 1973).

La perte des prophages n'a pas provoqué de changement des propriétés physiologiques importantes des souches telle que la vitesse d'acidification du lait qui pourrait avoir des répercussions en pratique industrielle. Ceci rejoint les observations de Georghiou, Phua et Terzaghi (1981) sur la souche R1.

En conclusion, l'état multilysogène fréquent chez les streptocoques lactiques semble rendre difficile l'élimination des prophages. Jusqu'à présent peu de souches ont été guéries et il a été difficile d'impliquer les prophages étudiés dans le déterminisme d'un caractère bactérien. Les seules observations dans ce sens sont les changements d'immunité de la souche TL933 et de sensibilité aux phages 
de la souche TL823. Par ailleurs, il est à craindre que les recombinaisons entre phages tempérés d'une même cellule puissent contribuer à l'apparition de nouveaux types de phages dans les usines.

\section{Remerciements}

Nous remercions Mmes A. Batt et A. Jaffe pour leurs conseils.

\section{Bibliographie}

Anderson (N.), Doane (F. W.) (1972), - Agar diffusion method for negative staining of microbial suspensions in salt solutions. Appl. Microbiol., 24, 495-496.

Bramucci (M. G.), KegGrns (K. M.), Lovett (P. S.) (1977). - Bacteriophage conversion of spore-negative mutants to spore-positive in Bacillus pumilus. J. Virol., 22, 194-202.

Chopin (M. C.), Снорin (A.), Roux (C.) (1976), - Definition of bacteriophage groups according to their lytic action on mesophilic lactic streptococci. Appl. Environ. Microbiol., 32, 741-746.

CHopin (M. C.), Rousseau (M.) (1983), - Tubular heads in bacteriophages from lactic streptococci. Appl. Environ. Microbiol., 45, 294-296.

Davies (F. L.), Gasson (M. J.) (1980). - Reviews of the progress of dairy science: genetics of lactic acid bacteria. J. Dairy Res., 48, 363-376.

Davies (F. L.), UNDERWood (H. M.), GASSON (M. J.) (1981). - The value of plasmid profiles for strain identification in lactic streptococci and the relationship between Streptococcus lactis 712, ML3 and C2. J. Appl. Bact., 51, 325-337.

Duval-IFlaH (Y.) (1972), - Lysogenic conversion of the lipase in Staphylococcus pyogenes group III strains. Can. J. Microbiol., 18, 1491-1497.

FreEMAN (V. J.) (1951). - Studies on the virulence of bacteriophage-infected strains of Corynebacterium diphteriae. J. Bacteriol., 61, 675.

Gasson (M. J.), Davies (F. L.) (1980). - Prophage-cured derivatives of Streptococcus lactis and Streptococcus cremoris. Appl. Environ. Microbiol., 40, 964-966.

Georghiou (D.), Phua (S. H.), Terzaght (E.) (1981). - Curing of a lysogenic strain of Streptococcus cremoris and characterization of the temperate bacteriophage. J. Gen. Microbiol., 122, 295-303.

Glover (S. W.), Schell (J.), Symonds (N.), Stacey (K. A.) (1963). - The control of host-induced modification by phage Pl. Genet. Res. Camb., 4, 480-482.

HEAP (H. A.), LAWRENCE (R.C.) (1977). - The contribution of starter strains to the level of phage infection in a commercial cheese factory. N.Z.J. Dairy Sci. Technol., 12, 213-218.

HeAP (H. A.), JARVIS (A. W.) (1980). - A comparison of prolate and isometric headed lactic streptococcal bacteriophages. N.Z.J. Dairy Sci. Technol., 15, 75-81.

Holloway (B. W.), Cooper (G. N.) (1962). - Lysogenic conversion in Pseudomonas aeruginosa. J. Bacteriol., 84, 1321-1324.

Huggins (A. R.), SANDine (W. E.) (1977). - Incidence and properties of temperate bacteriophages induced from lactic streptococci. Appli. Environ. Microbiol., 33, 184-191.

JARVIS (A. W.) (1982). - Multiple lysogeny in lactic streptococci, XXIth Int. Dairy Cong., Moscou, 1, 314. 
Kemp (C. L.), Howatson (A. F.), Siminovitch (L.) (1968). - Electron microscope studies of mutants of lambda bacteriophage. I. General description and quantitation of viral products. Virology, 36, 490-502.

KozaK (W.), RaJchert-Trzpil (M.), ZaJdel (J.). Dobrzanski (W. T.) (1973). - Lysogeny in lactic streptococci producing and not producing nisin. Appl. Microbiol., 25, 305-308.

Lawrence (R. C.), Thomas (T. D.), Terzaghi (B. E.) (1976). - Reviews of the progress of dairy science: cheese starters. J. Dairy Res., 43, 141-193.

Lawrence (R. C.), Heap (H. A.), Limsowtin (G.), Jarvis (A. W.) (1978). - Symposium: Research and development trends in natural cheese manufacturing and ripening. J. Dairy Sci., 61, 1181-1191.

LoWRIE (R. J.) (1974). - Lysogenic strains of group N lactic streptococci. Appl. Microbiol., 27, 210-217.

McKaY (L. L.), BaLdWIN (K. A.) (1973). - Induction of prophage in Streptococcus lactis C2 by ultraviolet irradiation. Appl. Microbiol, 25, 682-684.

McKay (L. L.), Baldwin (K. A.) (1974). - Simultaneous loss of proteinase-and lactose-utilizing enzyme activities in Streptococcus lactis and reversal loss by transduction. Appl. Microbiol., 28, 342-346.

Rettenmier (C. W.), Hemphill (H. E.) (1973). - Prophage mediated interference affecting the development of Bacillus subtilis bacteriophage $\Phi$ e. J. Virol., 11, 372-377.

Reyrolle (J.), Chopin (M. C.), Letellier (F.), Novel (G.) (1982.). - Lysogenic strains of lactic acid streptococci and lytic spectra of their temperate bacteriophages. Appl. Environ. Microbiol., 43, 349-356.

Terzaghi (B. E.), SANDiNe (W. E.) (1975). - Improved medium for lactic streptococci and their bacteriophages. Appl. Environ. Microbiol., 29, 807-813.

Terzaghi (B. E.), SANDINE (W.) (1981). - Bacteriophage production following exposure of lactic streptococci to ultraviolet radiation. J. Gen. Microbiol., 122, 305-311.

Trautner (T. A.), Pawlek (B.), Gunthert (U.), Canosi (U.), Jentsch (S.), Freund (M.) (1980). - Restriction and modification in Bacillus subtilis: identification of a gene in the temperate phage SP $\beta$ coding for a $B s u R$ specific modification methyltransferase. Mol. Gen. Genet., 180, 361-367. 